\title{
Ambiances
}

anbiances Environnement sensible, architecture et espace urbain Varia | 2015

\section{L'enfant des villes. Considérations sur la place du jeu et la créativité de l'architecte face à l'émergence de la ville garantie}

Child of the city: what place for game / play and architectural creativity facing guaranteed city's emergence

\section{Marc Breviglieri}

\section{OpenEdition}

\section{Journals}

Édition électronique

URL : http://journals.openedition.org/ambiances/509

DOI : 10.4000/ambiances.509

ISSN : 2266-839X

\section{Éditeur :}

Direction Générale des Patrimoines - DAPA - MCC, UMR 1563 - Ambiances Architectures Urbanités (AAU)

\section{Référence électronique}

Marc Breviglieri, «L'enfant des villes. Considérations sur la place du jeu et la créativité de l'architecte face à l'émergence de la ville garantie », Ambiances [En ligne], Varia, mis en ligne le 20 avril 2015 consulté le 19 avril 2019. URL : http://journals.openedition.org/ambiances/509; DOI : 10.4000/ ambiances.509

Ce document a été généré automatiquement le 19 avril 2019

\section{c)}

Ambiances is licensed under a Creative Commons Attribution-NonCommercial-NoDerivatives 4.0 International License. 


\title{
L'enfant des villes. Considérations sur la place du jeu et la créativité de l'architecte face à l'émergence de la ville garantie
}

\author{
Child of the city: what place for game / play and architectural creativity facing \\ guaranteed city's emergence
}

Marc Breviglieri

1 Que nous livre l'enfant qui sort dans la rue? Nous verrons que, jusqu'à un certain point, et d'une certaine façon, il vient nous rappeler l'inscription du vivant dans l'humanité de l'homme. Nous allons conduire la réflexion à partir d'un événement contingent au bruissement habituel des villes : le tout jeune enfant est tenu par la main de son parent qui insensiblement desserre son étreinte, et brutalement, il s'échappe. Il semble répondre à un impératif imaginaire et dessine l'expression d'une soudaine envolée aérienne, un "geste d'échappement ". Le trouble et parfois la réaction inquiète qui s'ensuivent soulèvent quelque chose de significatif au regard de la manière dont la ville peut laisser ouverts des espaces de potentialité (donc de jeu) et s'éprouver sous des tonalités affectives changeantes. Mais nous voudrions mettre aussi en tension cette possibilité même : la ville, du moins celle qui émerge des formes contemporaines de gouvernance urbaine, n'est-elle pas en train de perdre certaines de ses qualités sensibles, ruinant en un sens le foisonnement possible de ces espaces de jeu? En cherchant à garantir, dans son processus d'édification, des qualités objectives comparables qui la placent dans un registre de concurrence métropolitaine, et qui l'orientent insensiblement vers un style international standardisé et aseptisé, ne voit-elle pas disparaitre une part importante de ses dimensions expérientielles et de sa profondeur affective ? Et alors, certes l'enfant ne cessera pas de (vouloir) jouer et de s'amuser, mais il émerge nécessairement une interrogation critique sur ce qu'induit pour le (tout) jeune citadin l'appauvrissement des possibilités de jeu. Et parallèlement, regardant en direction de la conception architecturale des espaces urbains, on peut se questionner sur la place qui sera encore 
faite pour ces zones d'instabilité et de turbulence dont l'enfant est le porteur, pour la teneur débordante de son expérience sensible, pour ses gestes qui colorent subitement la texture de la vie urbaine ${ }^{1}$.

\section{Motricité de l'enfant, possibilités empiriques de jeux et première valeur d'usage de la ville}

2 Commençons par une scène qui marque l'antériorité chronologique à tout déplacement de l'enfant dans l'espace urbain: le parent l'emporte dans la rue, il le plonge dans une ambiance dont il ignore tout et il le tient très possiblement en sécurité dans ses bras. Il faut d'abord considérer le point de vue de l'effet d'impression que vit l'enfant. Pour appréhender la valeur inaugurale de cette expérience enfantine, nous allons édifier progressivement un jeu verbal qui tissera un ensemble de métaphores spatialisées par rapport à son référent corporel et impressionnel. Dans les bras du parent, l'enfant est donc porté en hauteur, il s'accroche au cou solide, il ne prête le flanc à aucune atteinte, il lui semble flotter dans les airs. Il est secoué par les pas qui progressent dans la ville mais qu'importe : il se fie entièrement au corps familier qui l'enveloppe dans son intimité et qui devient la voûte porteuse et rassurante d'un se laisser aller vers le monde du dehors. Mais en étant déplacé de la sorte, l'enfant n'a pas vraiment la possibilité de se ménager un accès aux choses alentour perceptibles par la vision, il retient une puissance d'exploration, il reste en-deçà de lui-même, à l'intérieur d'un rétrécissement momentané et contraint de ses pouvoirs moteurs. L'enfant, donc, est déplacé, il n'a pas de lui-même le pouvoir de changer de lieu, d'orienter le déplacement dans une direction; parfois il bat des pieds, il se contorsionne, il contracte un geste qui voudrait partir et répondre aux stimulations de cette ambiance largement nouvelle à ses yeux. Car au-dehors, les stimulations motrices de la ville s'ouvrent devant lui comme un éventail.

Placé dans une situation de relative passivité réceptrice, l'enfant n'en goûte pas moins un présent ambiant duquel se détache une atmosphère sensorielle et, goûtant, il prend déjà part à cette atmosphère à travers des gestes, plus ou moins expressifs, de « collage à la syntonie " (Csepregi, 2002). L'enfant dispose d'un caractère singulièrement impressionnable, il se laisse facilement emporter par ces «ennuagements atmosphériques » (Tellenbach, 1983). Et la rue se montre impressionnante à son goût : les pentes et les descentes, les surfaces glissantes et brillantes, des visages inconnus et le frôlement de l'étrange, le serpent épais de la foule, le flot grondant du trafic, les édifices grandioses blottis les uns contre les autres, des échafaudages surgissant de terre, une porte brutalement ouverte et derrière, une embrasure obscure. L'impressionnabilité de l'enfant, accrue par le fait qu'il est emporté dans un mouvement qu'il ne détient pas, se prête tout particulièrement à son inclinaison ludique. Particulièrement extensible, celleci n'a aucun mal à faire que la rue s'étende sous ses yeux à la manière d'un espace de potentialités diverses et de grandes variations phénoménales où se dresse un horizon infini de jeux variés. L'expérience naïve et immédiate où prolifèrent des paysages étonnants et des possibilités empiriques remarquables de jeux, constitue ainsi pour lui un pôle d'attraction intuitif. Mais il faut d'emblée considérer que cette expérience n'est pas exclusive. L'enfant est aussi pris par un vertige inverse au contact de la rue. Son étrangeté et son extériorité peuvent se condenser en une sensation de menace. La rue, parc à jeux sans limite, prend alors aussi l'aspect d'un monde hostile. 
Il demeure important de réunir cette polarité imaginaire des perceptions (parc à jeux / monde hostile) en cela qu'elle éveille une dialectique de l'attirance et de la crainte qui soulève une agitation émotionnelle remarquable. Il est d'ailleurs possible que cette ambivalence à la racine de l'existence affective soit finalement inséparable de la fascination troublante dont certaines villes détiennent le secret. Toutefois, cette agitation émotionnelle anime la vie de l'enfant de tout son pouvoir mystérieux : elle se traduit dans l'exaltation de la curiosité et dans l'excitation de l'imagination et de la fantaisie. Cette dissonance sensible n'est pas le terme mais le début du chemin hésitant d'un apprentissage depuis lequel des prises sont acquises sur le corps et, corrélativement, constituées pour cet espace impressionnant. L'enfant y introduit des formes motrices déjà éprouvées dans la quotidienneté de l'habitation, tout en produisant des mouvements apparentés qui cherchent à s'accommoder et à s'accorder au monde de la rue, conférant à la ville sa première valeur d'usage.

\section{Sens latent du geste d'échappement, trois configurations de la ville}

\section{Pénétrer une couche atmosphérique différente}

Revenons à l'événement sur lequel notre propos se tient posé : l'enfant est accompagné d'un parent dont l'emprise physique et la surveillance visuelle se relâchent insensiblement et là, profitant d'un serrement de main relâché ou d'une étreinte moins pressante, il part comme un jet, bondit pour un bref moment d'évasion. On doit entendre cette évasion comme un mouvement ascensionnel, l'enfant se sent pousser des ailes, il se dégage d'une pesanteur que l'inquiétude toute verticale du parent n'avait fait qu'accroître. Il s'engouffre alors brusquement dans une couche atmosphérique différente. La ville est là, dans cette première impression configurante: dans cet abord de la différence, dans cette excitation par l'étrangeté et ce goût pour l'étrange. L'enfant y prépare son geste superbe et orgueilleux de puissance: il s'échappe dans l'espace alentour en ouvrant un horizon de possibles. L'ivresse de l'échappée libère en même temps une décharge d'affects d'ordre vital et un tourbillon d'appétences. Ses vocalisations de joie, ses bras qui moulinent tous azimuts, sa course effrénée sont le témoignage de son triomphe.

\section{Sentir un espace vertigineux}

6 Ce geste d'échappement ne correspond véritablement ni à une manifestation précoce d'autonomie individuelle, ni à un refus adressé au parent dans la posture du défi. Il n'a de sens que sous la réserve d'une certaine approbation continument protectrice et soutenante, n'advient que sous la condition de l'existence préalable d'une solide enveloppe de bienveillance qui autorise la création d'espaces de potentialité, d'itinéraires explorateurs et finalement de régions ludiques où l'enfant met au contact du monde ses facultés motrices. L'enfant anxieux, au contraire de celui qui peut aisément s'《 extravertir » (Chavarot, 2004) dans les termes qui viennent d'être posés, a du mal à se livrer au monde, il a tendance à resserrer ces espaces du possible. Il peine en ce sens à libérer la nature excitante du geste d'échappement dans la mesure où elle dépend largement du jeu confiant soudain instauré à la lisière de cette enveloppe protectrice. On 
retrouverait sans mal la trace de cette excitation en regardant de près un ensemble de jeux d'enfant, notamment les jeux fondés sur un apparaître / disparaître, puis ceux dits de "chats» (Balint, 1972). Ces jeux sont entièrement animés et structurés par la possibilité d'un moment d'échappée qui implique et mobilise un puissant frisson suscité par la séparation soudaine d'un lieu de refuge sécurisant et la traversée subséquente d'un espace vide et potentiellement menaçant. L'enfant éprouve l'angoisse d'une perte de prise, d'un appui qui se dérobe et l'imminence incertaine de son retour dans l'affolement d'avoir été trop loin. Il se sera laissé happer un moment par un espace dénué d'ancrage affectif sécurisant, un espace vertigineux donc, semblable à ce qu'il vivait en apprenant à marcher lorsqu'il se jetait d'un objet stable à l'autre. Il semblait tanguer alors, entre deux récifs, dans la quête d'un bord où pouvoir se tenir, mettant au défi la chute et apprenant à se situer et se mouvoir entre le proche et le lointain. La ville se présente ici dans un second habit configurant, source d'autres élans de l'imaginaire et d'autres dynamiques spatialisantes. Elle n'est plus seulement pourvoyeuse d'une impression de différence atmosphérique, elle se montre vertigineuse, proposant du vide entre des bords : l'un attire, les autres attachent, et se maintient indéfiniment cette tension entre le proche et le distant, où foisonnent les intrigues de l'existence et se consument les énergies vitales².

\section{Toucher une matière confuse (malléable pour le jeu et durcie par les conventions)}

7 L'enfant qui s'échappe dans la rue cristallise un moment d'angoisse, il laisse derrière lui une confusion affective, soulève éventuellement l'inquiétude du parent qui alarme à son tour les autres passants. Il jette un trouble. L'espace finalisé (orienté vers un point, rythmé sur un rapport temps / distance) dans lequel circulait le parent (qui avait planifié de faire ses courses, se promener, rendre visite, prendre le bus, etc.) est désorganisé et remis en question: une direction imprévue s'est ouverte, un rythme s'est emballé. Chaque trouble est un désordre naissant pour celui qui le vit, à la fois une épaisseur viscérale et affective qui est remuée, un retentissement corporel qui infuse et nourrit l'émotion, et une direction de sens qui déjà se cherche et s'esquisse hors de l'expérience usuelle du monde familier. Le trouble déroute le passant, mais il est aussi ce par quoi la ville se rend émotionnellement tangible, ce par quoi elle se rencontre et s'« apprend " (Breviglieri \& Trom, 2003; Garcia Sanchez, Le Méner \& Yaro, 2009). Et le parent ou le proche qui veillait sur l'enfant génère et s'inscrit lui aussi dans un tel processus d'apprentissage : sa réaction, plus ou moins vive, sous-tend un mouvement d'éclairement de la structure normative (fonctionnelle et civile) du domaine public urbain. En resserrant l'émotion suscitée par le trouble autour des sentiments de mise en péril de l'enfant, de responsabilité civile et de respect pour le voisinage le plus proche, il dessine déjà un trajet instructif. A cet égard, si le parent, le proche ou le passant interviennent dans leur autorité d'éducateur, ce sera notamment pour mettre sous les yeux de l'enfant insouciant un monde assagi, ou plutôt aplati par l'utilisation raisonnable qui doit en être faite. Un monde tout d'abord signalé par le permis et le défendu, l'indiqué et le contreindiqué, puis organisé par des polarités normatives (propre / sale, inoffensif / dangereux, utile / inutile), bref, un monde objectif appelant à partager une atmosphère normative et à rentrer dans un certain ordre public partagé.

8 La ville est ici traversée par un nouveau sillon configurant, elle revêt une troisième signification profonde à partir de l'expérience d'échappement qui nous intéresse. Comme 
elle se prête au trouble qui infléchit toute univocité, et comme elle (s')expose au brouillage référentiel, à l'entrecroisement continu des référents subjectifs corporels et des référents nécessaires à une organisation spatiale collectivisée, elle demeure potentiellement confuse. Il faut y voir sa capacité propre à générer de l'équivoque, de l'embarras, des impressions opaques, des distances de sens et des écarts de perception. Elle représente donc un artifice humain fondamentalement labile et déformable : tantôt matière durcie autour des arêtes conventionnelles de la vie publique (qui entretiennent une certaine normalité de la coexistence) et autour des structures fonctionnelles et utilitaires (qui font sa masse calculable), tantôt matière souple et malléable se prêtant au relief flottant des espaces de jeu. Par ses gestes déroutants, l'enfant met à l'épreuve le sens trivialement fonctionnel de la ville, et plus largement ses dimensions spatiotemporelles moralisées. Mais en faisant trembler ses éléments structurels et ses principes d'ordonnancement, qu'il expose soudain dans le champ confus de l'apparaitre, il vient y puiser et y révéler sa substance la plus archaïque : il prend contact avec la volonté de vivre-ensemble et les sacrifices que sa mise à l'épreuve suppose.

\section{Genèse du goût de la ville}

Par ce geste d'échappement, il aura donc été permis à l'enfant de s'affirmer doublement, ou de grandir par deux fois. Une première fois en libérant un mouvement audacieux pour vibrer avec le monde de la rue, potentialisant une région ludique, donnant ainsi du volume au corps propre dans le tissu phénoménal déployé par le jeu et la vitalité débordante. Mais, par ailleurs, il aura aussi obtenu sa voluminosité du fait d'avoir pris de l'importance aux yeux d'autrui : il s'est montré capable d'affecter l'humeur de son parent (voire de troubler le public des passants) et il a fait se télescoper l'ambiance ludique, emportée par son geste, avec l'atmosphère normative du domaine public (Breviglieri, 2007)3. C'est précisément sur ce front de turbulence qu'il grandit une seconde fois : il lève sur son passage ce rayon normatif, on doit l'éclairer sur la nature de son geste, sur ses conséquences, sur la force d'obligation de la relation de confiance. Si son geste n'est pas instantanément oublié, ignoré ou réprimé, peut-être lui livrera-t-on une esquisse pédagogique de la bonne grammaire des relations en public. Alors, bien que frémissant encore de son envolée, il sera peut-être conduit de son sentiment de légèreté au sentiment de responsabilité; le parent consentant à dévorer un morceau d'enfance, l'enfant calmant ou maitrisant le bouillonnement des premiers pouvoirs du corps. Dans cette polarité tendue entre responsabilité et légèreté germe le goût de la ville où vient puiser l'étendue sensible et opulente de son expérience.

\section{Ce que l'architecte doit à l'enfant}

\section{Stimuler l'imaginaire anthropologique}

Comment cette ville qu'abrite l'enfant derrière ses gestes d'échappement éclaire-t-elle l'architecte dans sa tâche d'édification ? Une telle question suppose de donner à cette légère "note d'existence " ${ }^{4}$, à cette irruption soudaine de l'enfant dans l'ambiance de la ville, une dimension analytique qui ouvre d'elle-même de nouvelles possibilités de description dans le domaine de l'architecture. Ainsi, au prétexte d'un regard sur ce geste d'échappement, avons-nous observé la disposition de l'enfant à vouloir goûter ce qu'il 
voit, à être donc enveloppé par l'atmosphère sensorielle de la ville bien avant que de pouvoir la regarder de surplomb. Il y révèle, on l'a vu, son goût pour l'étrange et le différent, puis son goût pour le vertige qui est une quête de bords où pouvoir se tenir, enfin son goût pour le milieu confus et la malléabilité même de la matière urbaine. Par là, il permet, nous invitant à sa hauteur, de capter une manifestation de la ville, d'en révéler une texture particulière, de garder en vue un champ phénoménal qui la fait exister. C'est la texture de ce champ phénoménal que l'architecte ne sait peut-être plus lire lorsqu'il regarde la ville à la manière de l'adulte, en refoulant notamment cette disposition qu'il avait, enfant, à s'introduire dans le monde à la faveur de bondissements inquiets ou joyeux explorant de nouveaux espaces. Il la refoule ou la tient la plupart du temps en réserve dès lors qu'il n'y reconnaît plus ces prémisses émancipatoires qui donnent prise sur le monde, qu'il ne décèle plus à travers ces gestes d'enfant qu'un comportement néfaste ou du moins problématique au regard d'un certain ordre légitime. En l'occurrence : un comportement bruyant, dissipé, imprudent, accompagné par des excès de pulsions dont l'enfant doit se libérer en grandissant, et dont l'adulte cherche par conséquent à se différencier en affichant une contenance qui participe au reflet d'un espace public bien ordonné. C'est d'ailleurs en partie cette contenance en public qui confère à l'adulte une forme de souveraineté aux yeux de l'enfant.

11 Ainsi, l'adulte peine à reconnaître et à pénétrer l'atmosphère ludique dans laquelle l'enfant baigne pleinement; d'ailleurs, il tend à considérer l'enfance comme un paysage lointain et excentrique dont les pôles d'intérêt et les centres d'expérience ne le concernent plus directement. Mais par là, perdant de vue le champ d'appréhension sensible de l'enfant, et notamment sa prédilection pour les franges incertaines du jeu, l'architecte ne court-il pas le risque d'ôter à la ville une dimension anthropologique depuis laquelle se pense l'accueil des ressources que l'enfant nous aide à voir et à comprendre? Ce faisant, il contribuerait en un sens au délabrement ou à l'appauvrissement de la «ville plurielle» (Pattaroni, 2007), celle dont le telos serait de rester divisible et recomposable en une multiplicité d'horizons d'usage.

\section{Spatialiser l'attachement}

L'enfant invite donc l'architecte à se rendre attentif au caractère limité de ses propres conceptions anthropologiques touchant à l'usage des espaces urbains. S'il prenait appui sur l'observation des potentialités spontanées de l'enfant, travaillant imaginativement au bénéfice d'une ouverture perceptive vers une modalité d'expérience dont il a probablement oublié les mobiles profonds, il pourrait explorer une expression particulière de la ville et configurer un autre récit d'usage. L'architecte peut faire venir des éléments d'interprétation à partir des modes relationnels et manipulatoires que l'enfant tient en son pouvoir, et par la suite, tirer des lignes de pensée qui parlent de ce qui organise structurellement la ville comme «espace de contact» qui contribue à « former la personne humaine » (Choay, 2011). Or, si l'enfant divulgue quelque chose de la structure primordiale des villes, cela concerne en premier lieu une manière de les découvrir et de se lier à elles. Une manière procédant d'abord du corps propre, parce que la ville est ainsi, pour commencer, comme une substance sensible où le corps s'enfonce et se répand dans la nuance affective des choses. Et depuis ce point d'origine d'où peut s'étendre la résonance corporelle, sous l'incidence de la parution d'une altérité qui en exalte la tension, s'enclenche la dialectique du propre et du commun : les impulsions se 
libèrent mais restent susceptibles d'être apprivoisées, le geste d'exploration est aussi un mouvement d'appropriation, la curiosité finit par laisser une empreinte familière sur le lieu. C'est en cela que l'on s'attache à la ville, pour cela que ma mémoire est à jamais exposée à des récurrences affectives que je dois à mes plongées enfantines dans la rue.

L'attachement à la ville a peu de choses à voir avec ce qu'un vaste réseau d'acteurs intervenant dans sa conception et son aménagement désigne aujourd'hui comme les éléments composant son attractivité. Les critères d'attractivité de la ville (circulation fluide, mise en valeur patrimoniale, assortiment de commerces, services efficaces, etc.) sont là pour prétendre mesurer et garantir objectivement ce qui constitue sa qualité "normale", agissant au passage comme une puissante information marchande qui occasionne un effet de séduction et de captation des capitaux humains et financiers. La gouvernance urbaine en quête d'attractivité objective ne fait pas que placer la ville dans le vaste espace marchand globalisé où se met fortement en jeu sa réputation, elle alimente aussi la propagation massive des systèmes de normalisation et de certification de la qualité qui participe du capitalisme contemporain (Thévenot, 2014). Et sous l'influence de cette double dynamique, largement promue par la Communauté Européenne, les métropoles s'uniformisent tout en se rendant comparables. Subissant ce mouvement relatif d'uniformisation, la ville perd et se défait d'un ensemble de particularités sensibles où pouvaient s'ancrer les trajets diversifiés et les convictions intimes de l'attachement. C'est pourquoi, le projet de se conformer aux indicateurs d'attractivité, généralisé à l'horizon de l'urbanité contemporaine, pourrait bien menacer le caractère attachant des villes.

\section{Creuser les espaces transitionnels}

Prolongeons nos considérations au sujet de la main de l'architecte. En quoi, disions-nous, celle-ci se montre-t-elle redevable à l'enfant ? Ce que l'on doit à l'enfant, afin qu'il puisse s'exprimer dans un monde qui cherche à l'élever, c'est d'abord de le faire jouer (Stiegler, 2008). Et précisément, lui permettre de jouer dans la rue, c'est l'engager dans un jeu où la «bande d'espace potentiel» (Belin, 2001) qui lui est laissée correspond pour lui à un élargissement de la communauté des vis-à-vis. Il rencontre un ailleurs, il pénètre dans un dehors, il personnifie l'étranger, il dévêt de son angoisse l'inquiétude de l'autre, il extrait un moment ce dernier de ses seuls fantasmes pour ouvrir un espace de communication et ainsi, il dilate la surface de retentissement de ses propres expressions. Il la dilate en faisant vibrer la structure normative du bien et du mal, en se donnant l'occasion d'une anticipation affective et motrice des valeurs qui polarisent la communication, ici, les valeurs d'un monde commun en public (Breviglieri, 2014). Cette anticipation émerge du noyau primordial de l'imagination. Il semble qu'une ville ne dispose de profondeur sensible, ce en quoi elle se rend attachante, qu'en nourrissant un imaginaire contingent à sa découverte et à l'usage varié qui en est fait. Dans cette profondeur sensible se redessine indéfiniment la tâche d'ouvrir l'existence en commun sur un avenir indéterminé. C'est par là que la ville peut prétendre perpétuer la source de l'imaginaire, à la fois celle dont l'enfant a besoin pour jouer, et celle que l'homme politique réclame pour entretenir son art de gouverner. En ce sens, la main de l'architecte se retrouve dépositaire d'une mission architecturale vouée à creuser la profondeur sensible de la ville, à ouvrir par la même occasion des possibilités multiples d'engagement dans le monde commun. N'est-ce pas alors l'ampleur de la perspective anthropologique mise en jeu par cette profondeur, à 
travers la compréhension de ce par quoi l'homme se montre faillible et capable dans son usage du monde, qui en retour doit venir alimenter l'inspiration et maintenir une tension vivante sur la créativité architecturale?

Mais pour maintenir vivante cette tension créatrice, l'architecte a besoin de maintenir une confiance inaltérable dans ce que l'usage potentiellement ouvre, pluralise et déploie. Une confiance qui doit ainsi résister aux précarités de l'existence, aux surgissements des expériences négatives, à la confusion affective comme aux énigmes de la faillibilité. Car l'usage est fait de moments d'errance et de désorientation, de tâtonnement et d'hésitation, à partir desquels s'affirment son potentiel d'ajustement et son mouvement naturel d'appropriation. C'est de cette résistance aux écueils qu'il rencontre que l'usage tire son savoir-faire et devient un lieu d'expression pour la créativité. Or un défi global que rencontre aujourd'hui « l'agir humain » (Jonas, 1990), et notamment dans le registre du bâtir, repose sur la fragilité même de ce crédit accordé à la complexité inépuisable du monde et à la diversité des engagements humains qu'il rend possible. Cette défiance larvée appelle au déploiement foisonnant des autorisations légitimes et formelles à faire crédit, et c'est en partie ce développement qui alimente la croissance sans précédent de l'instrumentation visant à garantir la qualité normale de la ville, dont la politique de certification est la pointe avancée. On ne reviendra pas ici sur les raisons historiques de l'avènement généralisé d'une "culture de la suspicion » ou d'une "heuristique de la peur ", sur le fait même que la responsabilité engagée dans toute forme de projet fondationnel se retrouve, plus que jamais, liée à l'idée de dangerosité, à une posture d'aversion aux risques ou au besoin croissant d'élaboration de garanties sur la prévention de catastrophes (ibid.). On remarquera simplement que cette faille ouverte dans le crédit basique fait au monde et à l'humain, ce doute généralisé sur la viabilité du développement de notre habitation sur terre, se répercute sur l'édification d'une architecture urbaine qui voit proliférer, à tous les étages de la construction, un ensemble d'expertises, de prescriptions techniques normatives et de dispositifs de certifications de la qualité. C'est donc à ces experts qualifiés, à ces prescriptions certifiées et à ces dispositifs d'évaluation que le crédit est désormais censé être accordé.

\section{Contre l'enfant, l'appauvrissement expérientiel de la ville garantie}

Il est souvent question, derrière des objectifs toujours plus nombreux de mise en conformité aux normes règlementaires et d'obtention de labels de qualité, de faire valoir un consensus formé autour de la sécurité de l'enfant, dont il ne faut pas oublier qu'il est aussi là pour réduire les risques de responsabilité des parents et des aménageurs. On vient de souligner aussi que cette quête de certifications et de labels de qualité a le pouvoir de garantir un accès à l'espace marchand concurrentiel et qu'elle contribue donc largement à la régulation du capitalisme contemporain mondialisé. Mais concrètement, ce consensus signale aussi un net changement qualitatif de l'espace public auquel l'enfant peut avoir accès. D'un côté la démultiplication d'aires de jeux sécurisées semble consacrer une division spatiale qui n'autorise l'enfant à jouer que dans ces seuls territoires délimités. Cette division impose une rupture de continuité entre les régions ludiques qui animent quotidiennement son existence et l'espace public de la rue. Ce dernier tend alors irrémédiablement à perdre ses propriétés d'espace potentiel et transitionnel (Winnicott, 2002), lieu majeur d'apprentissage où l'enfant expérimente le dehors de l'habitation et, 
d'une certaine façon, s'entraîne à grandir. De l'autre côté, mais non sans liens, en voulant apporter toutes les garanties de sécurité pour l'enfant, l'aménagement de la rue peut induire un appauvrissement expérientiel considérable pour ce dernier. Au lieu d'être un champ d'expérience corporelle incitatif, lieu d'invention de nouveaux usages comme de nouvelles relations à autrui, elle est d'abord conçue pour faciliter la surveillance parentale et renforcer les probabilités d'utilisation raisonnable du domaine public. Il faudrait, à cet égard, mentionner le triple registre de forces induit par une architecture qui intervient sur la matérialité de l'espace en (1) dissuadant de certaines actions, (2) guidant les gestes et (3) canalisant des parcours. La garantie alors obtenue sur l'utilisation raisonnable et fonctionnelle des espaces publics urbains signifie, simultanément, qu'une liberté d'usage s'amoindrit. Et derrière cela, s'opère le choix implicite d'évacuer les possibilités d'irruption du jeu qui, précisément, ont le pouvoir de déborder le script inscrit dans la fonction et de déjouer la prédictibilité de l'utilisation raisonnable, en mobilisant le monde alentour de manière inattendue, provocatrice et insouciante.

C'est notamment pour de telles raisons, et sous l'empire autoritaire de ce processus normalisant et de ce consensus sécuritaire auxquels contribue involontairement l'enfant, que le paysage de la rue s'aménage et qu'il s'homogénéise à l'échelle de toutes les métropoles occidental(isé)es. On la débarrasse des éléments urbains dont les propriétés sont rendues floues à l'usage et qui permettent encore la formation d'espaces de jeu : recoins et angles aigus, irrégularités du sol, surfaces élimées ou terreuses, parcelles en friche, rebords saillants et surfaces d'appui en hauteur, mobilier instable ou déformé, autant de prises qui permettent à l'enfant de se cacher, de glisser, de se balancer, de s'agripper, de sauter, etc. A l'horizon discutable d'une inquiétude excessive, on pourrait dire que chacune de ces propriétés génère bien un risque pour la sécurité de l'enfant car la complexité motrice, rythmique et directionnelle des mouvements impliqués dans le jeu potentialisent une mésaventure (déséquilibre, chute, accrochage, strangulation, coincement, infection, etc.). Mais alors, chemin faisant, l'architecte, placé sous le patronage d'une telle inquiétude de garantie des choses, se rend responsable d'anéantir précisément ce qui enchantait l'enfant dans la ville et, du même coup, de négliger la profondeur intime d'une conviction enfantine selon laquelle il faut jouer pour vivre bien. Il abolit ce qui faisait de l'espace public urbain un espace transitionnel où l'enfant pouvait explorer un monde en jouant, il contribue à le rendre enclin à ne plus vouloir sortir au dehors de l'habitation pour pouvoir s'y amuser.

S'affirme donc à l'échelle internationale l'édification d'une "ville garantie », une ville qui voudrait donner l'assurance certaine de la qualité de ses propriétés et qui privilégie pour cela un gouvernement par les normes et la certification (Thévenot, 1997, 2006). Ville garantie qui, en un sens, se défie et se détourne d'un crédit premier donné à notre " condition d'humanité imprévisible » porteuse d'un chaos fertile (Giannini, 1992), et qui bouleverse l'ordre ouvert des choses en cherchant d'abord à donner la ferme assurance des termes de leur appropriation et de leur utilisation. Ville garantie, donc, qui appauvrit la spatialité constituante de la ville en rétrécissant les champs affectifs et les complexités émotionnelles qui s'éprouvent à son contact. Ville garantie, ville lisse et sans anfractuosités, dans laquelle je ne serai jamais plus sollicité par cette trouble curiosité qui me pousse à m'enfoncer en elle. Son destin empirique s'est semble-t-il coupé du noyau imaginaire qui tient en germe une ville ouverte aux climats électriques et incertains, aux potentialités latentes d'investissement de l'espace, à la lucide créativité de l'usage et aux engagements variés du propre et du commun qui alimentent une substance en tension ${ }^{5}$. 
Pour mettre en valeur cette substance en tension, l'enfance nous a donné matière à description. Et en particulier son geste d'échappement que nous répercute la rue, cette soudaine éruption existentielle qui parfois trouble son entourage, ce jeu créant l'espace dans lequel l'enfant a fait de la place pour grandir, cette tentation aérienne et provocatrice que partage l'imaginaire utopique.

\section{BIBLIOGRAPHIE}

Balint, Michael. 1972. Les voies de la régression. Paris : Payot.

Belin, Emmanuel. 2001. Une sociologie des espaces potentiels. Logique dispositive et expérience ordinaire. Bruxelles : De Boeck Supérieur.

Bonnet, Aurore. 2013. Qualification des espaces publics urbains par les rythmes de marche. Approche à travers la danse contemporaine. [en ligne]. Thèse de doct. Grenoble : Université de Grenoble / Urbanisme mention Architecture. Consultée le 29 octobre 2014. Url : https://tel.archivesouvertes.fr/tel-00846710v2/document.

Breviglieri, Marc. 2007. Ouvrir le monde en personne. Une anthropologie des adolescences. In : Breviglieri, Marc \& Cicchelli, Vincenzo (eds.). Adolescences méditerranéennes. L'espace public à petits pas. Paris : L'Harmattan. p. 19-59.

Breviglieri, Marc. 2013. Une brèche dans la « ville garantie »? Architectures d'usage et espaces intercalaires. In : Cogato Lanza, Elena ; Pattaroni, Luca ; Tirrone, Barbara \& Piraud, Mischa (eds.). De la différence urbaine. Le quartier des Grottes / Genève. Genève : MétisPress. p. 213-236.

Breviglieri, Marc. 2014. La vie publique de l'enfant. Participations. Revue de sciences sociales sur la démocratie et la citoyenneté. $n^{\circ}$ 9, p. 97-123.

Breviglieri, Marc \& Trom, Danny. 2003. Troubles et tensions en milieu urbain. Les épreuves citadines et habitantes de la ville. In : Cefaï, Daniel \& Pasquier, Dominique (eds.). Les sens du public : publics politiques et médiatiques. Paris : PUF / CURAPP. p. 399-416.

Charles, Julien. 2012. Comment la cartographie méconnaît les habitants. Le formatage de la participation dans une commune belge. Participations. Revue de sciences sociales sur la démocratie et la citoyenneté. $\mathrm{n}^{\circ} 4, \mathrm{p} .155-178$.

Chavarot, Jean-Marc. 2004. Les directions de sens du toucher. In : Chamond, Jeanine (eds.). Les directions de sens. Phénoménologie et psychopathologie de l'espace vécu. Argenteuil : Le Cercle Herméneutique. p. 93-116.

Choay, Françoise. 2011. La terre qui meurt. Paris : Fayard.

Csepregi, Gabor. 2002. Le jeu rituel. Contribution à une phénoménologie de la mémoire corporelle. Etudes phénoménologiques. $\mathrm{n}^{\circ}$ 36, p. 97-118.

Garcia Sanchez, Pedro ; Le Méner, Erwan \& Yaro, Ibrahim. 2009. Les mondes des Bacoramans. Etude interactionniste sur les enfants de rue à Ouagadougou. Ouagadougou / Paris : Samu Social International / Union Européenne. 
Giannini, Humberto. 1992. La « Réflexion » quotidienne. Vers une archéologie de l'expérience. Aix-enProvence : Ed. Alinea.

Jonas, Hans. 1990. Le principe responsabilité. Une éthique pour la civilisation technologique. Paris : Ed. du Cerf.

Merleau-Ponty, Maurice. 1994 [1945]. Phénoménologie de la perception. Paris : Gallimard.

Pattaroni, Luca. 2007. La ville plurielle : quand les squatters ébranlent l'ordre urbain. In : Bassand, Michel ; Kaufmann, Vincent \& Joye, Dominique (eds.). Enjeux de la sociologie urbaine. Lausanne : PPUR. p. 283-314.

Ricœur, Paul. 1950. Philosophie de la volonté. Tome 1. Paris : Aubier.

Soulier, Nicolas. 2012. Reconquérir les rues. Paris : Ulmer.

Stiegler, Bernard. 2008. Prendre soin. 1. De la jeunesse et des générations. Paris : Flammarion.

Tellenbach, Hubertus. 1983. Goût et atmosphère. Paris : PUF.

Thévenot, Laurent. 1997. Un gouvernement par les normes. Pratiques et politiques des formats d'information. In : Conein, Bernard \& Thévenot, Laurent (eds.). Cognition et information en société. Paris : Ed. de l'EHESS (Raisons Pratiques nº). p. 205-241.

Thévenot, Laurent. 2006. L'action au pluriel. Sociologie des régimes d'engagement. Paris : La Découverte.

Thévenot, Laurent. 2014. Nouvelles autorités à l'épreuve de la critique. Le gouvernement par l'objectif. In : Frère, Bruno (ed.). Le tournant de la théorie critique. Paris : Desclée de Brouwer. p. 216-235.

Thibaud, Jean-Paul \& Duarte, Cristiane Rose (eds.). 2013. Ambiances urbaines en partage. Pour une écologie sociale de la ville sensible. Genève : MétisPress.

Winnicott, Donald Woods. 2002 [1945]. Jeu et réalité. L'espace potentiel. Paris : Gallimard.

\section{NOTES}

1. Ce texte se situe à l'entrecroisement de deux essais récents; l'un portant sur les transformations contemporaines du gouvernement de l'urbain (Breviglieri, 2013), l'autre touchant aux premiers contacts de l'enfance avec la vie en public (Breviglieri, 2014). Il tire largement profit d'une série d'observations et de prises de vue filmiques réalisées sur les espaces publics d'une ville marocaine (Asilah) et d'un quartier populaire de Lisbonne. Cette investigation a pu alimenter récemment, à Genève, un événement pédagogique lié à la reproduction réfléchie d'une ambiance urbaine étalée sur vingt-quatre heures (As Ruas de Lisboa. Couleurs lisboètes et sonorités lusophones). Cet événement a bénéficié de la participation du vidéaste Laurent Valdès et de l'artiste et designer Camille Dedieu. Enfin, je tiens à remercier Emmanuelle Tricoire pour la discussion que nous avons eue concernant le travail descriptif et notionnel entrepris dans ce texte.

2. J'indique, bien que le sujet de l'enfance n'y apparaisse pas directement, l'analyse originale de la marche dans l'espace urbain proposée par Aurore Bonnet dans sa thèse de doctorat (Bonnet, 2013). Cette dernière fait intervenir de manière judicieuse une réflexion sur la danse qui introduit aux thèmes du rapport à la gravité et de l'élévation, ou de l'appui et de la chute, permettant d'intégrer fortement des contenus signifiants vitaux et existentiels dans la compréhension du déplacement. 
3. Sur le thème de la voluminosité du corps propre, voir notamment le troisième chapitre de la première partie de la Phénoménologie de la perception (Merleau-Ponty, 1994).

4. L'expression est de Paul Ricœur (1950).

5. Nous n'abordons pas ici les différentes modalités à travers lesquelles se dessinent des formes pratiques usuelles ou soudaines d'oppositions plus ou moins politisées à la ville garantie ; que ces oppositions fassent vibrer autrement les espaces en partage (un ensemble de contributions recoupent ce point d'analyse dans: Thibaud \& Duarte, 2013) ou qu'elles instaurent des " architectures d'usage" (Breviglieri, 2013) permettant de "reconquérir autrement la rue » (Soulier, 2012). Une ethnographie pointant les abus de l'instrumentation visant à garantir la qualité normale de la ville est aussi intéressante afin d'étayer en amont notre point de vue (Charles, 2012).

\section{RÉSUMÉS}

La présence des tout jeunes enfants dans la rue se manifeste parfois comme des gestes d'évasion et d'exploration arrachés à la surveillance parentale. A y regarder de près, la manifestation inspirée de ces " gestes d'échappement " semble dévoiler une dimension essentielle de la relation sensible au milieu urbain que l'architecte ne devrait en rien perdre de vue. L'enfant peut faire exister la ville autrement et en révéler une texture particulière que l'adulte tend à ne plus savoir lire. Mais cette ouverture au champ phénoménal de l'enfant, qui aide au travail imaginaire de l'architecte, subit une tension de sens opposé venant d'un ensemble de directives et de prescriptions techniques normatives liées au développement d'une politique de certification de la qualité particulièrement active dans le domaine de l'enfance et du design urbain. En voulant garantir l'utilisation raisonnable et informée de la ville, cette politique évacue bon nombre de possibilités d'irruption du jeu dans l'espace public, grâce auxquelles l'enfant apprend à vivre avec d'autres, potentialise des capacités remarquables et trouve parfois un incomparable lieu d'enchantement.

The presence of young children in the street sometimes manifests itself through moves to escape or explore beyond parental supervision. Taking a closer look, the inspired manifestation of such gestures of escape reveals an essential dimension of the sensory relation to the urban environment, which architects should bear in mind. A child can make the city exist differently, revealing a particular texture which an adult often no longer registers. But this opening into the child's phenomenal field, which may help architects in their efforts of imagination, is subject to a contrary tension due to an array of normative technical directives and constraints entailed by the spread of quality-certification policies, particularly forcible with regard to children and urban design. In their drive to guarantee reasonable, informed use of the city, such policies eliminate many of the loopholes through which play may break out into the public space, thus depriving children of the opportunity to learn to live with others, potentialize remarkable abilities and, perhaps, discover a place of unique delight. 
INDEX

Mots-clés : rue, design urbain, enfant, jeu / ludique, apprentissage, usage, atmosphère(s), trouble(s), phénoménologie, vie, affectivité

Keywords : street, urban design, child, game - play / fun, learning, use, atmosphere(s), trouble (s), phenomenology, life, affectivity

\section{AUTEUR}

\section{MARC BREVIGLIERI}

Les thèmes de recherche de Marc Breviglieri touchent aux configurations et aux aménagements variés de l'habitation humaine, aux apprentissages de la vie commune, aux dimensions liant corps et espace et enfin aux questions d'ordre affectif, éthique et politique posées par l'expérience du soin. Il développe une sociologie d'inspiration phénoménologique nourrie par l'ouverture de perspectives méthodologiques liées à l'image (photographie-vidéo). Il est Professeur à la Haute école spécialisée de Suisse occidentale (HETS Genève), et chercheur associé au CRESSON (UMR 1563 AAU).

https://ies-geneve.academia.edu/MarcBreviglieri marc.breviglieri@hesge.ch 